\title{
A comparative study of multi-objective optimal power flow based on particle swarm, evolutionary programming, and genetic algorithm
}

\author{
Solmaz Kahourzade • Amin Mahmoudi • \\ Hazlie Bin Mokhlis
}

\begin{abstract}
This paper compares the performance of three population-based algorithms including particle swarm optimization (PSO), evolutionary programming (EP), and genetic algorithm $(\mathrm{GA})$ to solve the multi-objective optimal power flow (OPF) problem. The unattractive characteristics of the cost-based OPF including loss, voltage profile, and emission justifies the necessity of multi-objective OPF study. This study presents the programming results of the nine essential single-objective and multi-objective functions of OPF problem. The considered objective functions include cost, active power loss, voltage stability index, and emission. The multiobjective optimizations include cost and active power loss, cost and voltage stability index, active power loss and voltage stability index, cost and emission, and finally cost, active power loss, and voltage stability index. To solve the multiobjective OPF problem, Pareto optimal method is used to form the Pareto optimal set. A fuzzy decision-based mechanism is applied to select the best comprised solution. In this work, to decrease the running time of load flow calculation, a new approach including combined Newton-Raphson and Fast-Decouple is conducted. The proposed methods are tested on IEEE 30-bus test system and the best method for each objective is determined based on the total cost and the convergence values of the considered objectives. The pro-
\end{abstract}

S. Kahourzade $(\bowtie) \cdot$ A. Mahmoudi

UM Power Energy Dedicated Advanced Centre (UMPEDAC),

University of Malaya, Kuala Lumpur, Malaysia

e-mail: solmazkahourzade@gmail.com

A. Mahmoudi

e-mail: amaminmahmoudi@gmail.com

H. B. Mokhlis

Department of Electrical Engineering, University of Malaya,

Kuala Lumpur, Malaysia

e-mail: hazli@um.edu.my gramming results indicate that based on the inter-related nature of the objective functions, a control system cannot be recommended based on individual optimizations and the secondary criteria should also be considered.

Keywords Evolutionary programming - Genetic algorithm · Multi-objective optimal power flow · Particle swarm $\cdot$ Pareto optimal method

\section{Introduction}

Optimal power flow (OPF) was introduced for the first time by Carpentier in 1962 [1] and developed later by Dommel and Tinney [2]. The aim of OPF is to optimize particular objective via adjustment of the power system control variables, while various equality and inequality constraints of the power system are satisfied. In the past two decades, OPF became one of the interest areas based on its capability of energy management. OPF is a large-scale, non-convex, highly constrained, and nonlinear problem with continuous and discrete variables [3]. Classical methods like linear programming (LP) [4], nonlinear programming (NLP) [5], quadratic programming (QP) [6], and interior point method (IM) [7] have been applied to solve OPF. However, the mentioned methods have difficulty in handling several local minima due to the non-convex nature of OPF and sensitivity to the starting point. Gradient-based methods such as Newton method solve the convergence problem; however, inequality constraints may not be satisfied [8]. To overcome the shortcomings of the classical methods, evolutionary methods are introduced including genetic algorithm (GA) [9], evolutionary programming (EP) [10], particle swarm optimization (PSO) [11,12], simulated annealing (SA) [13], differential algorithm (DE) [14], shuffle frog leaping algorithm (SFLA) [15], 
biogeography-based method [16], and artificial bee colony (ABC) algorithm [17].

Cost minimization was the primary objective of the OPF problem, but fuel cost increment and environmental concerns led to considering new objectives including active and reactive loss minimization, voltage stability index, and emission minimization. Therefore, multi-objective OPF was introduced to optimize a group of objective functions simultaneously $[18,19]$. Different multi-objective optimization methods like multi-objective evolutionary algorithm (MOEA) [20], multi-objective stochastic search technique (MOSST) [21], strength Pareto evolutionary algorithm (SPEA) [22], Niched Pareto genetic algorithm (NPGA) [23], non-dominated sorting genetic algorithm (NSGA) [24], multi-objective differential evolution (MODE) [25], and Tabu search method [26] have been proposed.

Although various multi-objective solutions for OPF problem have been proposed, there are a few systematic comparisons that only consider limited objective functions [2729]. Moreover, most of the conducted studies have been focused on merely one technique and alternative methods are copied from the referenced publications. Hence, the precision of results is vague due to the difference in the CPU execution time as well as the different level of details in the variables, constraints, and parameters of the alternative codes.

Therefore, this paper presents a comprehensive comparison based on the results of PSO, EP, and GA to solve multiobjective OPF for nine important objective functions including single-objective functions and multi-objective functions. Pareto optimal method is applied to create a set of solutions instead of one answer for PSO, EP, and GA. Finally, fuzzy decision-based mechanism is applied to select the best comprised solution of Pareto set for each one of the three methods. The considered single-objective functions include cost, active power loss, voltage stability index, and emission. The multi-objective functions include cost and active power loss, cost and voltage stability index, active power loss and voltage stability index, cost and emission, and finally cost, active power loss, and voltage stability index. Multi-objective optimal power flow is highly time consuming based on the multiple executions determined by Pareto-optimal solutions. In this work to decrease the running time of load flow calculation, a new approach including combined Newton-Raphson and Fast-Decouple is conducted. To verify the effectiveness of the proposed methods, IEEE 30-bus test system is taken as an example. This study provides a comprehensive comparison among PSO, EP, and GA to introduce the best method for solving a multi-objective OPF problem considering various objective functions. The selection of the optimizing method is based on the total cost and the convergence values of the considered objectives. To provide a reliable benchmark, all the mentioned methods have been programmed by the authors.
Therefore, the results presented in this paper for various methods are consistent, as the simulation is performed with the same computer and the level of details in the variables and constraints are unified. Thus, this paper provides knowledge for any researcher, graduate student, and practising engineer to get a good idea regarding the above-mentioned optimization methods.

The paper is organized as follows: Sect. 2 describes the problem constraints, formulation and objective functions; Sect. 3 demonstrates PSO, EP, and GA methods as well as the experimental values of parameters; Sect. 4 explains the proposed multi-objective optimization strategy; Sect. 5 presents the results and discussion; and finally Sect. 6 concludes the work.

\section{Optimal power flow formulation}

OPF determines the optimal settings of the power system network variables in a manner that the equality and inequality constraints are satisfied. OPF constraints and objective functions can be formulated as follows:

\subsection{Constrains}

Active and reactive power generations are defined as:

$$
\begin{aligned}
P_{g i}= & P_{d i}+V_{i} \sum_{j=1}^{n} V_{j}\left[G_{i j} \cos \left(\delta_{i}-\delta_{j}\right)\right. \\
& \left.+B_{i j} \sin \left(\delta_{i}-\delta_{j}\right)\right] \\
Q_{g i}= & Q_{d i}+V_{i} \sum_{j=1}^{n} V_{j}\left[G_{i j} \sin \left(\delta_{i}-\delta_{j}\right)\right. \\
& \left.-B_{i j} \cos \left(\delta_{i}-\delta_{j}\right)\right]
\end{aligned}
$$

where $P_{d i}$ and $Q_{d i}$ are the active and reactive power demand, $G$ and $B$ are the conductance and susceptance from Y-matrix, $V$ and $\delta$ are the magnitude and angle of the voltage, and $n$ is the number of buses. The nonlinear and functional operating constraints of the power flow are mathematically defined as:

$$
\left\{\begin{array}{l}
f(x, u) \\
g(x, u)=0 \\
h(x, u) \leq 0
\end{array}\right.
$$

where $g(x, u)$ is the typical load flow equation; $h(x, u)$ is the system operating constraints; $u$ is the vector control variable including voltage level, active power generated except at the slack bus, reactive power injected, and transformer tap changing; $x$ is the dependant variable vector including slack bus power, load bus voltage, reactive power generator outputs, and transmission line loading. In the power system network, the following constraints should be satisfied: 
1. Generator constraints:

$$
\begin{aligned}
& P_{g i}^{\min } \leq P_{g i} \leq P_{g i}^{\max } \\
& Q_{g i}^{\min } \leq Q_{g i} \leq Q_{g i}^{\max } \\
& V_{i}^{\min } \leq V_{i} \leq V_{i}^{\max }
\end{aligned}
$$

where $P_{g i}^{\max }, P_{g i}^{\min }, Q_{g i}^{\max }, Q_{g i}^{\min }, V_{i}^{\max }$, and $V_{i}^{\min }$ are the maximum and minimum values of the active power generation, reactive power generation, and voltage.

2. Shunt VAR constraint:

$Q_{c i}^{\min } \leq Q_{c i} \leq Q_{c i}^{\max }$

where $Q_{c i}^{\max }$ and $Q_{c i}^{\min }$ are the maximum and minimum shunt VAR compensations.

3. Transformer tap setting constraint:

$T_{i}^{\min } \leq T_{i} \leq T_{i}^{\max }$

where $T_{i}^{\max }$ and $T_{i}^{\min }$ are the maximum and minimum tap values.

4. Security constraints:

$$
\begin{aligned}
& M V A f_{l i} \leq M V A f_{l i}^{\max } \\
& V_{l i}^{\min } \leq V_{l i} \leq V_{l i}^{\max }
\end{aligned}
$$

where $M V A f_{l i}^{\max }$ is the maximum rating transmission; $V_{l i}^{\max }, V_{l i}^{\min }$ are the maximum and minimum line voltages.

\subsection{Objective functions}

Case 1: Generation cost objective

$F_{1}(x)=\sum_{i=1}^{N_{\text {gen }}} a_{i} P_{g i}^{2}+b_{i} P_{g i}+c_{i} \quad \$ / \mathrm{h}$

$P_{g i}$ is active power generated; $N_{\text {gen }}$ is the total number of generation units; $a_{i}, b_{i}$, and $c_{i}$ are the cost coefficients.

Case 2: Active power loss objective

$F_{2}(x)=\sum_{k=1}^{n l} g_{k}\left[V_{i}^{2}-V_{j}^{2}-2 V_{i} V_{j} \cos \left(\theta_{i}-\theta_{j}\right)\right] \quad \mathrm{MW}$

where $n l$ is the number of transmission lines, $\theta_{i}$ and $\theta_{j}$ are the bus voltage angles at the two ends of the $k$ th line, and $V_{i}$ and $V_{j}$ are bus voltage amplitude at the two ends of the $k$ th line.
Case 3: Voltage stability index objective

Voltage stability can be achieved by minimizing the voltage stability indicator $L$-index value for each bus [30]. The value of $L$-index varies from 0 to 1 during no-load to voltage collapse conditions, respectively.

$$
\begin{aligned}
L_{j} & =\left|1-\sum_{i=1}^{N_{\text {gen }}} F_{j i} \frac{V_{i}}{V_{j}}\right| j=N_{\text {gen }}+1, \ldots, n \\
F_{j i} & =-\left[Y_{L L}\right]^{-1}\left[Y_{L G}\right]
\end{aligned}
$$

where $\left[\mathrm{Y}_{L L}\right]$ and $\left[\mathrm{Y}_{L G}\right]$ are sub-matrices of the $Y$ bus matrix. The network equations in terms of the node admittance matrix are simply written as:

$I_{\text {bus }}=Y_{\text {bus }} V_{\text {bus }}$

For computing the voltage stability index value, it is necessary to cluster all nodes into two categories that involve load buses and generator buses as follows:

$\left[\begin{array}{c}I_{L} \\ I_{G}\end{array}\right]=\left[\begin{array}{ll}Y_{L L} & Y_{L G} \\ Y_{G L} & Y_{G G}\end{array}\right]\left[\begin{array}{l}V_{L} \\ V_{G}\end{array}\right]$

$I_{L}=Y_{L L} \times V_{L}+Y_{L G} \times V_{G}$

According to the superposition principle, the voltage $V_{L}^{k}$ in the load bus $k$ can be calculated as:

$$
\begin{aligned}
V_{L}^{k} & =\sum_{i=1}^{N_{\text {gen }}}\left(\left(Y_{L L}\right)^{-1} Y_{L G}\right)_{k, j} \times V_{G}^{k} \\
L_{j} & =\sum_{i=1}^{N_{\text {gen }}}\left(\left(Y_{L L}^{\prime}\right)^{-1} Y_{L G}\right)_{k, j}
\end{aligned}
$$

The $L$-indexes is computed for all the buses and the maximum $L$-index gives the proximity of the system to voltage collapse. For stable situations, the condition $0 \leq L_{j} \leq 1$ must not be violated for any of the nodes $j$. Hence, a global indicator $L$ that describes the stability of the whole system is given as:

$F_{3}(x)=L=\max \left(L_{j}\right)$

Case 4: Emission objective

$F_{4}=\sum_{i}\left(\alpha_{i} P_{g i}^{2}+\beta_{i} P_{g i}+\gamma_{i}+\xi_{i} \exp \left(\psi_{i} P_{g i}\right)\right)$ ton $/ \mathrm{h}$

where $\alpha_{i}, \beta_{i}, \gamma_{i}, \xi_{i}$, and $\psi_{i}$ are the emission coefficients.

The multi-objective functions are defined as:

Case 5: Fuel cost and active power loss

Case 6: Fuel cost and voltage stability index

Case 7: Active power loss and voltage stability index

Case 8: Fuel cost and emission

Case 9: fuel cost, active power loss, and voltage stability index 


\section{Optimization methods}

3.1 Particle swarm optimization

Particle swarm optimization is first proposed by Kennedy and Eberhart [31]. PSO is developed based on food-searching pattern of swarms like fishes and birds. The main advantages of PSO over other evolutionary methods are fewer parameter requirements as well as shorter calculation time. PSO is a population-based method consisting of particles with changing position with time in a search space. The position of the particles changes based on the experience of each particle and its neighbor particles. Consider the $n$-dimensional vectors $X_{i}=\left(x_{i 1}, x_{i 2}, \ldots, x_{i n}\right)$ and $V_{i}=\left(v_{i 1}, v_{i 2}, \ldots, v_{i n}\right)$ as the position and velocity of particle $i$, respectively. Modified velocity is defined as:

$V_{i}^{t+1}=\omega V_{i}^{t}+c_{1} \lambda_{1} \times\left(P_{i}^{t}-X_{i}^{t}\right)+c_{2} \lambda_{2} \times\left(g_{\text {best }}^{t}-X_{i}^{t}\right)$

where $P_{i}^{t}$ is the best position of particle $i, \omega$ the inertia weight parameter, $c_{1}, c_{2}$ the acceleration factors, $\lambda_{1}, \lambda_{2}$ the random values between 0 and 1 , and $g_{\text {best }}$ is the best neighboring position. The next position of each particle $X_{i}^{t+1}$ is calculated as follows:

$X_{i}^{t+1}=X_{i}^{t}+V_{i}^{t+1}$

Inertia weight parameter $\omega$ sets the balance between global and local search. Its value is calculated as given below:

$\omega=\left(\omega_{1}-\omega_{2}\right)\left(\frac{\text { iter }_{\max }-\text { iter }}{\text { iter }}\right)+\omega_{2}$

where $\omega_{1}, \omega_{2}$, iter, and iter $_{\max }$ represent the initial and final inertia weight, the iteration number, and the maximum number of iterations, respectively. It is important to set a suitable value to the maximum velocity of the particle. By adjusting a too large value to it, the previous best position may be missed, while a small value may lead to incomplete search in the search space. By adjusting the small values to the acceleration factors, particles may be trapped in a region far from the target spot, while the large acceleration factors cause sudden movement to the target region and consequently passing from the target region. The experimental values of PSO parameters that are applied in this study are presented in Table 1 [33].

\subsection{Evolutionary programming}

Evolutionary programming was first proposed in 1960 by Fogel [32]. It is a random search method that finds the optimum answer by evolving a population with a certain number of generations. EP highlights the behavior similarity between
Table 1 The values of PSO experimental parameters

\begin{tabular}{ll}
\hline PSO parameter & Value \\
\hline$c_{1}$ & 2 \\
$c_{2}$ & 2 \\
$\omega_{1}$ & 0.9 \\
$\omega_{2}$ & 0.4 \\
iter $_{\text {max }}$ & 200 \\
Swarm size & 60 \\
\hline
\end{tabular}

Table 2 The values of EP experimental parameters

\begin{tabular}{ll}
\hline EP parameter & Value \\
\hline Population size & 20 \\
Maximum generation & 200 \\
Constant $a$ & 0.95 \\
\hline
\end{tabular}

the parents and offsprings. The major steps for evolutionary programming-based optimal power flow are listed as follows:

- Initialize the initial population is generated with random value in a feasible range based on the information delivered from the buses data, lines data, generators data, shunts data, and transformer tap settings data.

- Mutation new offspring is generated by applying a Gaussian random variable with zero mean and standard deviation added to the existing chromosome.

- Competition and selection selection is based on the fitness value. The best individuals with maximum fitness values are selected to be parents of the next generation. The process of creating off-spring and selecting those with maximum fitness are repeated until there is no appreciable improvement in the maximum fitness value or it is repeated up to a pre-specified number of iterations.

Experimental values of EP' parameters that are used for programming in this paper are presented in Table 2 [33].

\subsection{Genetic algorithm}

Genetic algorithm is a general optimization algorithm, inspired from phenomena found in nature. The GA uses Darwin's principles to find the optimal formula for prediction or pattern matching. At each generation, a new set of approximations is created by applying a selection method to individuals based on their fitness level. The new generations are created by using crossover and mutation operators $[34,35]$. The GA consists of a population of strings transformed by the three genetic operators: selection, crossover and mutation. The only difference between EP and GA is the crossover step which is the main genetic operator and 
Table 3 The values of GA experimental parameters

\begin{tabular}{ll}
\hline GA parameter & Value \\
\hline Crossover probability & 0.9 \\
Mutation probability & 0.01 \\
Population size & 20 \\
Maximum generation & 200 \\
String length & 41 \\
\hline
\end{tabular}

consists of swapping chromosome parts between individuals. Crossover is not performed on each pair of individuals and it is frequently controlled by the crossover probability [36]. In crossover, the exchange of parents' information produces an offspring. As opposed to mutation, crossover is a rare process that resembles a sudden change to an offspring. This is performed via random selection of one chromosome from the population and then arbitrarily changing a part of its information. The benefit of mutation is that it randomly introduces new genetic material to the evolutionary process, perhaps thereby avoiding stagnation around the local minima. Experimental values of GA parameters are presented in Table 3 [33].

\section{Multi-objective solution}

Multi-objective optimization is developed to simultaneously optimize a set of objective functions while the governing constraints and equations are satisfied. Pareto optimal method using PSO, EP, and GA methods are developed to optimize multi-objective optimization including cost and active power loss, cost and voltage stability index, active power loss and voltage stability index, cost and emission, and finally cost, active power loss, and voltage stability index objectives. The process of multi-objective OPF is described as follows:

\subsection{Fuzzy model}

Firstly, the fuzzy model is applied to normalize the objectives of the multi-objective problem by applying membership functions. Membership function is determined considering upper and lower boundary values as:

$$
\lambda_{i}(X)=\left\{\begin{array}{ll}
1 & \text { if } F_{i}(X) \leq F_{i}^{\min } \\
\frac{F_{i}^{\max }-F_{i}(X)}{F_{i}^{\max }-F_{i}^{\min }} & \text { if } F_{i}^{\min }<F_{i}(X)<F_{i}^{\max } \\
0 & \text { if } F_{i}(X) \geq F_{i}^{\max }
\end{array}\right\}
$$

$F_{i}^{\min }, F_{i}^{\max }$ are delivered based on the optimization of each objective function. The maximum value of $F(X)$ for the multi-objective problem is considered the optimum value, while $F(X)$ is calculated as:

$$
F(X)=\min \left[\lambda_{1}(X), \lambda_{2}(X), \lambda_{3}(X), \ldots\right]
$$

4.2 Pareto optimal method

The next step is to apply Pareto optimal method to create a set of solutions instead of one answer for the multi-objective OPF problem. This method is based on the dominance concept that vector $X_{1}$ dominates vector $X_{2}$ while the following conditions are satisfied:

$$
\begin{gathered}
\forall i=\{1,2, \ldots, P\} F_{i}\left(X_{1}\right) \leq F_{i}\left(X_{2}\right) \\
\exists j \in\{1,2, \ldots, P\} F_{j}\left(X_{1}\right)<F_{j}\left(X_{2}\right)
\end{gathered}
$$

where $P$ is the number of control variables. Fuzzy method should be applied whenever objective functions are not in the same range.

\subsection{Fuzzy decision maker}

Finally, fuzzy decision maker is used to save non-dominated solutions in each iteration. It is defined as [37]:

$N_{\mu j}=\frac{\sum_{k=1}^{N_{\text {obj }}} \beta_{k} \times \mu_{k}(j)}{\sum_{j=1}^{o} \sum_{k=1}^{n} \beta_{k} \times \mu_{k}(j)}$

where $\beta_{k}$ is the weight factor for the $k$ th objective functions which is selected by the operator based on the importance of the objective functions. $N_{\mathrm{obj}}$ is the number of objective function, and $o$ is the number of non-dominated solutions. The optimum solution is the one with the maximum membership function $N_{\mu j}$.

Figure 1 shows the flowchart of the overall multi-objective OPF applied in this paper. The application of each evolutionary method is independent and the simulation is executed for PSO, EP and GA, separately.

\section{Results and discussion}

The proposed multi-objective algorithms using PSO, EP, and GA are tested on IEEE 30-bus test system. The single-line diagram of the IEEE 30-bus system is presented in the appendix. The values of the fuel cost coefficients and emission coefficients are listed in Table 4. To provide a systematic comparison, the base values of cost, loss, voltage stability index, and emission presented in Table 5 are considered [15]. The proposed work was implemented in MATLAB R2011, a computing environment with Intel(R) Xeon(R), $3.10 \mathrm{GHz}$ computer with 8 GB RAM.

The problem is initially solved as a single-objective optimization using PSO, EP, and GA methods. To decrease

\section{Link to Full-Text Articles:}

\section{http://link.springer.com/article/10.1007/s00202-014-0307-0}

